\title{
Erratum to: Simultaneously Measuring Lateral and Vertical Forces with Accurate Gap Control for Clarifying Lubrication Phenomena at Nanometer Gap
}

Kenji Fukuzawa $\cdot$ Kosei Hayakawa

Kazuki Matsumura $\cdot$ Shintaro Itoh ·

Hedong Zhang

Published online: 26 June 2013

(c) Springer Science+Business Media New York 2013

Erratum to: Tribol Lett (2010) 37:497-505

DOI 10.1007/s11249-009-9545-0

In the original version of this paper, the author's name, Noriki Matsumura, was incorrect. The correct name of the author is Kazuki Matsumura.

The online version of the original article can be found under doi:10.1007/s11249-009-9545-0.

K. Fukuzawa $(\bowtie) \cdot$ K. Hayakawa $\cdot$ K. Matsumura $\cdot$ S. Itoh ·

H. Zhang

Department of Micro/Nano Systems Engineering,

Nagoya University, Furo-cho, Chikusa-ku, Nagoya-shi,

Aichi 464-8603, Japan

e-mail: fukuzawa@nuem.nagoya-u.ac.jp 